\title{
Complete Plasmodium falciparum liver-stage development in liver-chimeric mice
}

\author{
Ashley M. Vaughan, ${ }^{1}$ Sebastian A. Mikolajczak, ${ }^{1}$ Elizabeth M. Wilson, ${ }^{2}$ Markus Grompe, ${ }^{3}$ \\ Alexis Kaushansky, ${ }^{1}$ Nelly Camargo, ${ }^{1}$ John Bial, ${ }^{2}$ Alexander Ploss, ${ }^{4}$ and Stefan H.I. Kappe ${ }^{1,5}$ \\ ${ }^{1}$ Seattle Biomedical Research Institute, Seattle, Washington, USA. ${ }^{2}$ Yecuris Corp., Tualatin, Oregon, USA. ${ }^{3}$ Oregon Stem Cell Center, \\ Papé Family Pediatric Research Institute, Oregon Health and Science University, Portland, Oregon, USA. ${ }^{4}$ Center for the Study of Hepatitis C, \\ The Rockefeller University, New York, New York, USA. ${ }^{5}$ Department of Global Health, University of Washington, Seattle, Washington, USA.
}

\begin{abstract}
Plasmodium falciparum, which causes the most lethal form of human malaria, replicates in the host liver during the initial stage of infection. However, in vivo malaria liver-stage (LS) studies in humans are virtually impossible, and in vitro models of LS development do not reconstitute relevant parasite growth conditions. To overcome these obstacles, we have adopted a robust mouse model for the study of $P$. falciparum LS in vivo: the immunocompromised and fumarylacetoacetate hydrolase-deficient mouse (Fab ${ }^{-/}, \mathrm{Rag}^{-/-}, \mathrm{Il}_{\mathrm{rg}} \mathrm{r}^{--}$, termed the FRG mouse) engrafted with human hepatocytes (FRG huHep). FRG huHep mice supported vigorous, quantifiable $P$. falciparum LS development that culminated in complete maturation of LS at approximately 7 days after infection, providing a relevant model for LS development in humans. The infections allowed observations of previously unknown expression of proteins in LS, including $P$. falciparum translocon of exported proteins 150 (PTEX150) and exported protein-2 (EXP-2), components of a known parasite protein export machinery. LS schizonts exhibited exoerythrocytic merozoite formation and merosome release. Furthermore, FRG mice backcrossed to the NOD background and repopulated with huHeps and human red blood cells supported reproducible transition from LS infection to blood-stage infection. Thus, these mice constitute reliable models to study human LS directly in vivo and demonstrate utility for studies of LS-to-blood-stage transition of a human malaria parasite.
\end{abstract}

\section{Introduction}

Plasmodium falciparum is the most deadly of the human malaria parasites. The disease continues to be a global health crisis and causes more than 250 million new clinical cases annually, resulting in over 800,000 deaths, mostly of children in sub-Saharan Africa (1). Female anopheline mosquitoes introduce infectious sporozoites into the host dermis when taking a blood meal. Sporozoites exit the bite site by migration, enter a blood vessel, and are carried to the liver (2). Here, each sporozoite traverses numerous hepatocytes before it invades a final hepatocyte with the formation of a parasitophorous vacuole (PV) (3). Ensconced in the PV, the parasite undergoes liver-stage (LS, also called exoerythrocytic form [EEF]) development, culminating in the formation and release of tens of thousands of first generation merozoites (4). This preerythrocytic phase of the parasite life cycle is asymptomatic, and all clinical pathologies are caused by the ensuing asexual erythrocytic stage of infection. The erythrocytic stages are routinely studied in vitro, made possible by the development of a continuous culture system that allows asexual parasite replication in human $\mathrm{rbc}$ (hurbc) (5). However, studying the biology and pathophysiology of $P$. falciparum in vivo is difficult and is hampered by the lack of adequate animal models. Sporogonic stages are generated by feeding female Anopheles mosquitoes on in vitro gametocyte cultures, allowing progression of the parasite life cycle in the mosquito and subsequent sporozoite accumulation in the mosquito salivary glands. However, the P. falciparum LS has proven much more dif-

Authorship note: Ashley M. Vaughan and Sebastian A. Mikolajczak contributed equally to this work.

Conflict of interest: Markus Grompe is a shareholder of the Yecuris Corp. Citation for this article: J Clin Invest. 2012;122(10):3618-3628. doi:10.1172/JCI62684. ficult to study even though complete LS development in primary human hepatocytes (huHeps) and subsequent transition to an in vitro erythrocytic infection was shown over 25 years ago (6). Primary huHeps can be obtained both freshly isolated and cryopreserved from a variety of sources, but are in limited supply, do not proliferate in vitro, and are variable in their ability to support $P$. falciparum LS development. A huHep cell line (HC-04) that allows for complete $P$. falciparum LS development has been reported, although in this cell line, the rate of infection is low: $0.07 \%$ (7). Furthermore, complete LS development is difficult to obtain in vitro because it requires more than 7 days of culture and conditions are often not commensurate with extended culture periods. As a result of the aforementioned difficulties, few data on human malaria parasite LS biology are available. Conversely, rodent malaria models of LS development, both Plasmodium berghei and Plasmodium yoelii, are routinely used to study LS. These studies have led to a wealth of knowledge on LS biology, including development, differentiation, host/parasite interactions, and organelle replication as well as the effects of gene deletions on LS development (8).

Fluorescent P. yoelii parasites have been generated (9) and used to obtain LS transcriptomes and proteomes following the isolation and manipulation of infected hepatocytes by FACS (10), and thus, a wealth of data are available from which predictions of cellular and metabolic pathways for LS development can be inferred. For example, access to the LS proteome led to the hypothesis that de novo fatty acid synthesis would be essential to the development of LS, and this has indeed been shown by functional studies (11, 12). Nevertheless, there are clearly unique aspects to $P$. falciparum LS biology. Rodent malaria LS development takes just over 2 days, whereas $P$. falciparum LS development takes a week. Additionally, $P$. falciparum LSs express proteins that do not have rodent malaria 
orthologs such as LS antigen 1 (LSA1) (13). Furthermore, studies have shown that syntenic Plasmodium genes do not always share the same biological function in P. falciparum and rodent malaria models (14). Thus, it is imperative to develop robust small animal models for $P$. falciparum that allow analysis of various aspects of LS biology in vivo.

Recent advances in the use of immunocompromised mice engrafted with huHeps enabled the creation of human liver-chimeric mice. In vivo P. falciparum LS development has been shown in one of these models, the SCID mouse homozygous for the urokinase-type plasminogen activator transgene $(u \mathrm{~Pa})$ under the albumin promoter $(A l b)$ : the SCID Alb-uPA mouse, engrafted with huHeps $(15,16)$. The SCID Alb-uPA huHep mouse has also been successfully used to study hepatitis B (17) and hepatitis C (18) and was used more recently to study the attenuation of a $P$. falciparum parasite that fails to complete LS development (19). However, due to chronic liver disease, the adult mice only weigh about 10-12 grams, and the neonates suffer bleeding problems (20), making the SCID Alb-uPA mouse extremely challenging to work with. The mice must be bred as heterozygotes, and transplantation with huHep must take place right before weaning to rescue the fatal phenotype. Furthermore, the Alb-uPA transgene can revert, leading to repopulation of the liver with mouse hepatocytes. To overcome the obstacles associated with the SCID Alb-uPA mouse (21), we have utilized a different and more robust mouse model that can be repopulated with huHep, the immunocompromised and fumarylacetoacetate hydrolase-deficient mouse ( $\mathrm{Fah}^{-/-}, \mathrm{Rag}^{-/-}$, $\mathrm{Il}_{2 \mathrm{rg}^{--}}$, termed the FRG mouse) as well as the FRG mouse backcrossed to the NOD background (FRG NOD).

The FRG mouse is a triple gene knockout $(22,23)$. $R$ denotes recombination-activating gene 2 (Rag2), and G denotes IL-2 recep-

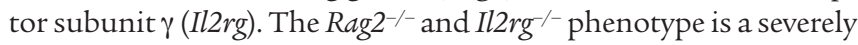
immunocompromised mouse lacking B, T, and NK cells that does not reject xenotransplanted huHeps. The $F$ denotes fumarylacetoacetate hydrolase (Fah). Due to the lack of FAH, the hepatocytes of $\mathrm{Fah}^{-/-}$mice suffer buildup of intracellular fumarylacetoacetate, resulting in their death. The phenotype is ablated by the addition of 2-(2-nitro-4-trifluoromethylbenzoyl)-1, 3-cyclohexanedione (NTBC) to the mouse diet (24). FRG mice implanted with huHeps (FRG huHep) are cycled with NTBC, which allows for the repopulation of the mouse liver with huHeps when regulated destruction of the mouse hepatocytes occurs by NTBC withdrawal. Repopulation levels can reach in excess of $90 \%$. Unlike the SCID Alb-uPA mouse, the FRG mice are healthy, breed as homozygotes, and are free of liver damage while dosed with NTBC. Furthermore, huHep that have proliferated in a donor FRG mouse can be serially transplanted into many recipient mice, which allows for the rapid expansion of animals repopulated with isogenic hepatocytes (22). Recent studies with FRG huHep mice have shown that they can be used for studies of infection with both hepatitis B and hepatitis C viruses (25).

We have successfully infected FRG huHep mice with $P$. falciparum sporozoites and observed LS developmental progression to full maturation, exoerythrocytic merozoite formation, and merosome release over a 7-day period. Furthermore, FRG mice backcrossed to the NOD background and repopulated with huHeps and hurbc supported complete LS development and a reproducible transition to blood-stage infection. Blood-stage infections were subsequently maintained in continuous culture. Our results show that the FRG huHep and FRG NOD huHep mouse models of $P$. falciparum LS development will be a robust tool to further study the biology of human LS parasites. Furthermore, the ability to transition P. falciparum from LS-to-blood-stage infection allows complete life-cycle progression in the laboratory. This will accelerate fundamental studies, such as parasite genetic crosses, in a small animal model.

\section{Results}

P. falciparum in vivo LS development in the FRG huHep mouse. The FRG huHep mice (all female) used in this study were obtained from the Yecuris Corp., and the huHep repopulation index of the mouse liver was estimated based on the levels of human serum albumin. Repopulation of all mice used in the study ranged between $60 \%$ and $90 \%$ (data not shown). Sporozoites used for the study were generated in Anopheles stephensi mosquitoes that had been fed on mature gametocyte cultures of $P$. falciparum NF54 parasites or, in one instance, the GFP-expressing NF54HT-GFP parasite. Mice were injected intravenously with sporozoites and sacrificed 3, 5, 6, and 7 days after infection, at which time liver tissue was collected for transcriptional analysis, histological evaluation and indirect immunofluorescence assays (IFAs).

IFAs on infected liver sections demonstrated that $P$. falciparum LS developed in the FRG huHep mice. At day 3 after infection, spherical LS were detected using a circumsporozoite protein (CSP) antibody, which localizes to the parasite surface and, as expected, was expressed in a circumferential pattern (Figure 1A). Using an antibody specific for human FAH, it was evident that P. falciparum LS always grew within the huHeps (Figure 1A). DAPI staining showed between 4 and 8 nuclear centers on day 3 of LS, indicating that parasite DNA replication was progressing. At day 5 after infection, LS had further grown and maintained their near spherical shape. The parasites were specifically labeled with an antibody to exported protein-2 (EXP-2) (26) in a pattern indicative of PV localization (Figure 1B). EXP-2 was previously localized to the PV membrane (PVM) of blood stages (27). More recently, EXP-2 was shown to be part of the P. falciparum translocon of exported proteins (PTEX) (28), a translocon that is believed to be responsible for the trafficking of exported parasite proteins into the erythrocyte cytoplasm. A further protein component of the translocon is PTEX150, and interestingly, we also detected expression of this protein at 5 days of LS development (Figure 1B). Its localization was similar to that seen for EXP-2 (Figure 1B). Thus, components of the translocon are present in LS and are localized to the PV. Day 5 LS still expressed CSP (Figure 1B), the transcript of which was also detected at this time point in the SCID Alb-uPA huHep mouse LS infections (16). Day 5 LS also expressed PF10_0164 (ETRAMP10.3) (Figure 1B) in the PVM, the syntenic ortholog of the rodent malaria parasite gene coding for UIS4 (14). At day 7 after infection, we observed large multinucleated LS schizonts that had displaced the surrounding tissue. The LS were readily detected with EXP-2 and merozoite surface protein 1 (MSP1) antibodies (Figure 1, C and D). Codetection with the FAH antibody clearly showed the LS contained within a vastly expanded huHep with only remnant cytoplasm and a single nucleus pushed to the periphery of the infected cell (Figure 1C). The pattern of EXP-2 expression ceased to be circumferential, resulting in a complex internal pattern of staining as seen in Figure 1C, likely due to EXP-2 expression being associated with the PV lumen at this developmental stage. The PVM markers EXP-1 and EXP-2 did not overlap in late LS parasites, indicating that EXP-1 remained in the PVM during late LS development, but EXP-2 did 
A

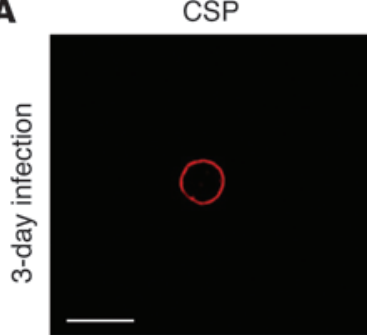

$\mathrm{FAH}$

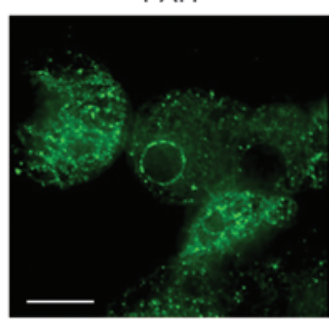

DNA

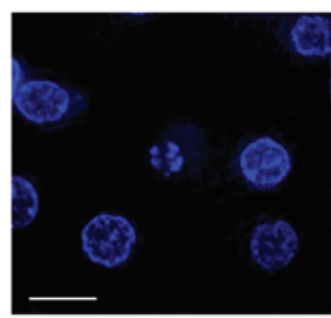

Merge

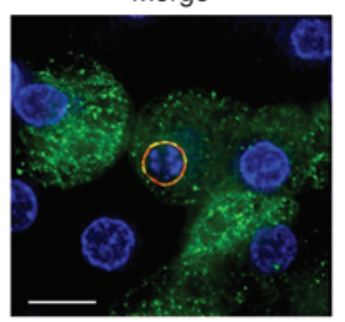

B

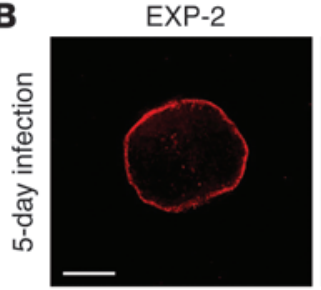

PTEX150

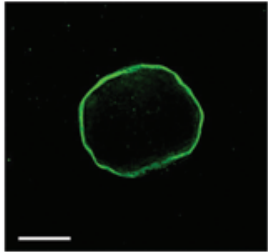

DNA/merge
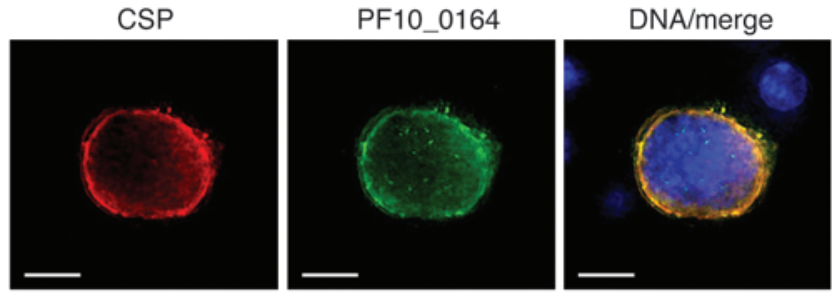

C
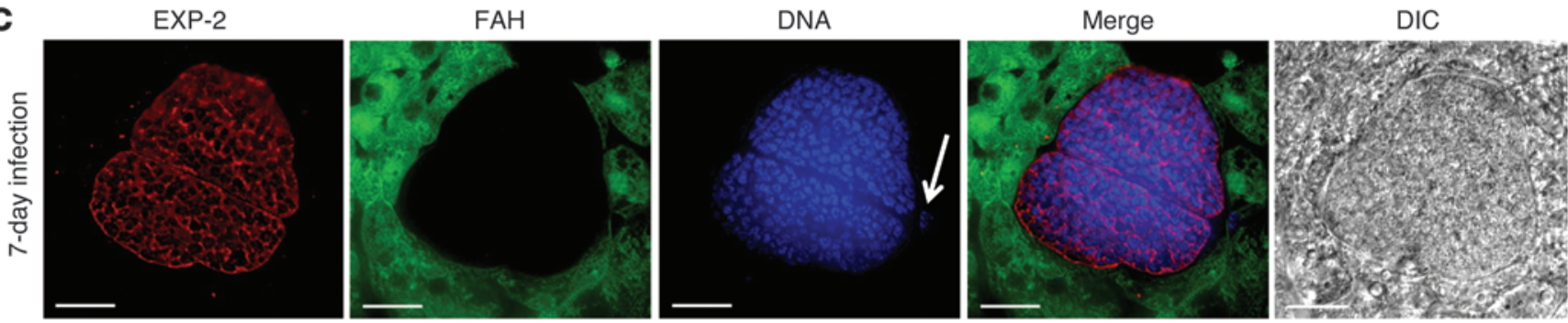

D
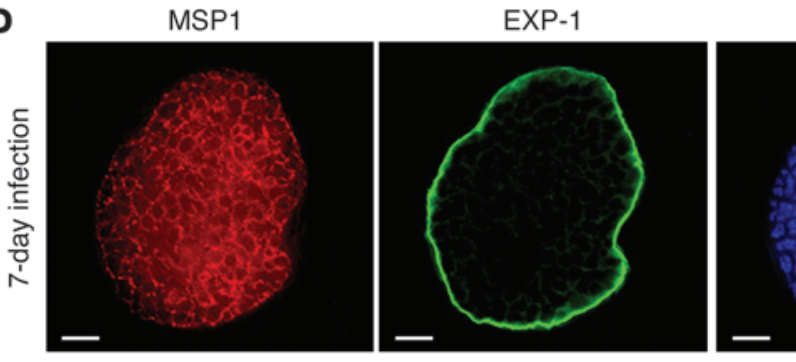

DNA

Merge

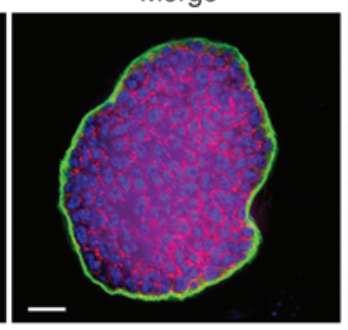

DIC

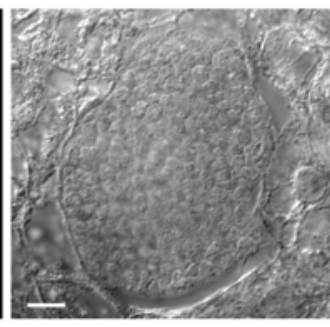

DNA

Merge

E

MSP1

$\mathrm{FAH}$
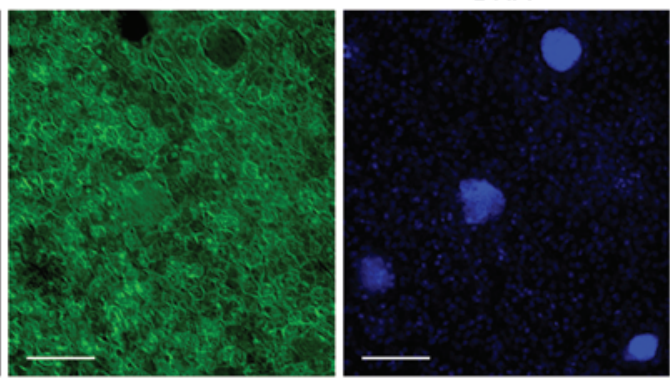

Figure 1

P. falciparum LS development in FRG huHep mice. Infected liver sections were assayed by indirect immunofluorescence using antibodies specific to $P$. falciparum for parasite detection. (A) LSs at day 3 of infection were visualized using antibodies to parasite CSP, which localizes to the parasite surface. (B) LSs at day 5 of infection were visualized with antibodies to EXP-2 and PTEX150, components of the Plasmodium translocon of exported proteins (28), which were both robustly expressed (3 panels on the left), as well as the PVM protein PF10_0164 (14) and CSP (3 panels on the right). LSs at day 7 of infection were visualized with antibodies to EXP-2 (C), MSP1 (E), and in combination with MSP1 and EXP-1 (D). huHeps were visualized with antibody to human FAH in A, C, and E, and the liver sections were visualized by differential interference contrast microscopy (DIC) in $\mathbf{C}$ and $\mathbf{D}$. DNA was visualized with DAPI in all panels. Note the nucleus of the infected hepatocyte in $\mathbf{C}$, which has been pushed to the extremity of the infected hepatocyte (white arrow in the DNA panel). Scale bars: $10 \mu \mathrm{m}(\mathbf{A}, \mathbf{B}$, and D); $20 \mu \mathrm{m}(\mathbf{C}) ; 100 \mu \mathrm{m}(\mathbf{E})$. 


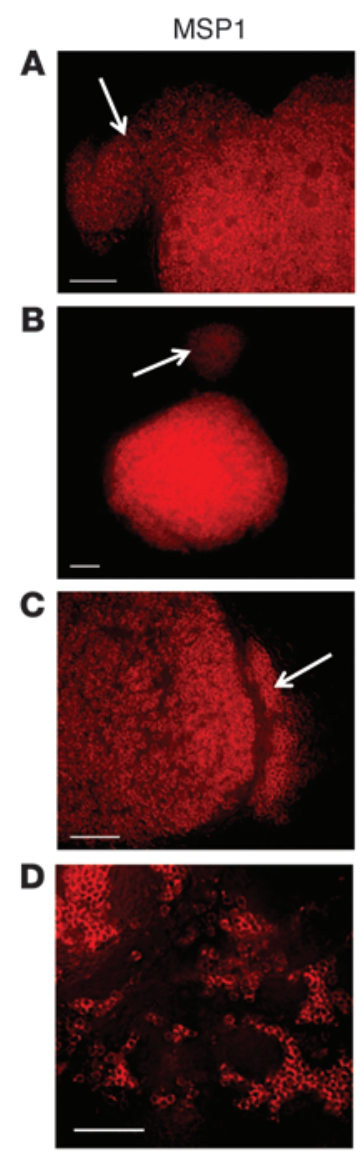

MSP1

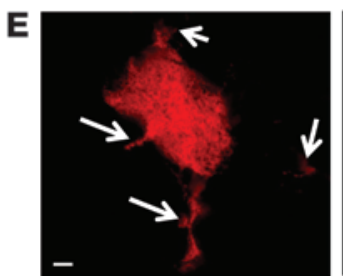

DNA
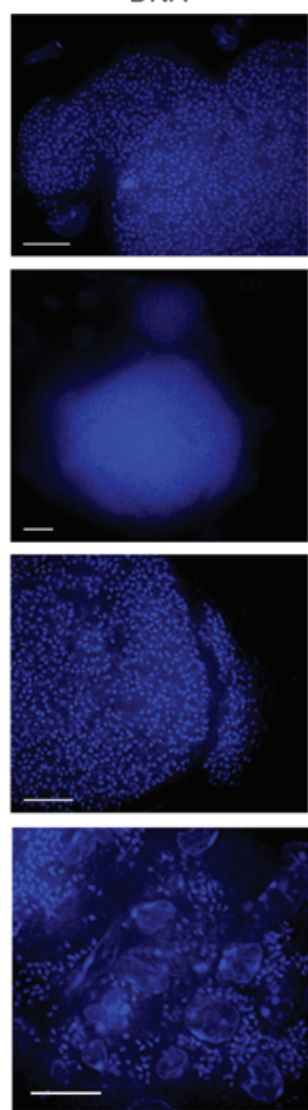

EXP-1

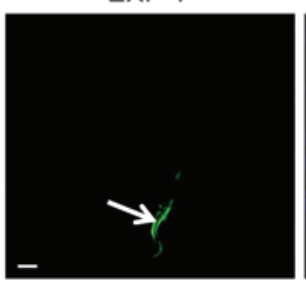

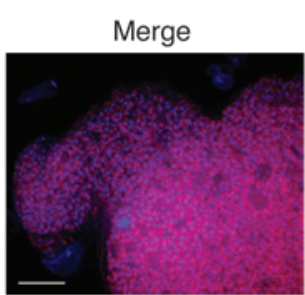
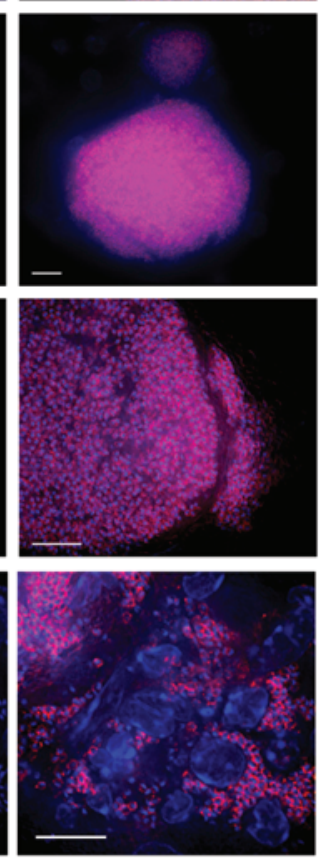

DNA

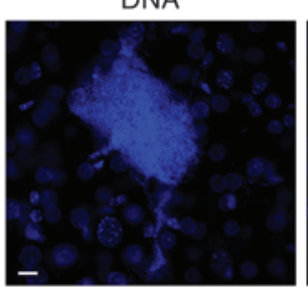

DIC
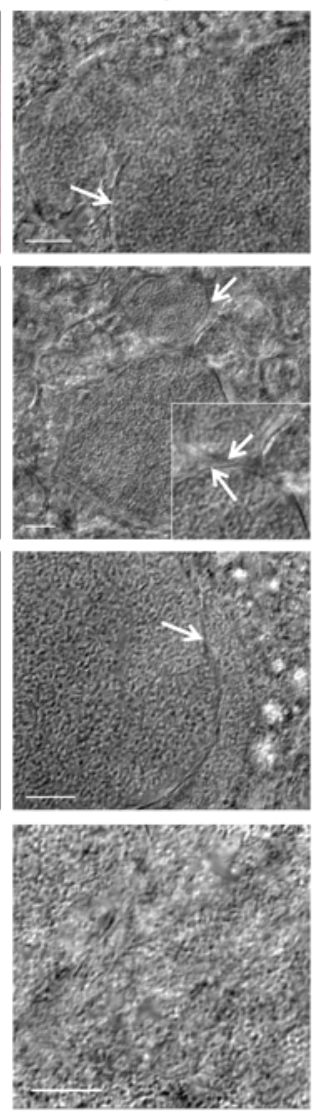

Merge

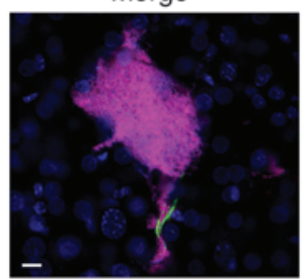

DIC

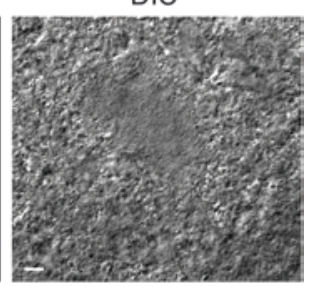

\section{Figure 2}

Maturation of $P$. falciparum LSs and exoerythrocytic merozoite release in FRG huHep mice. Indirect immunofluorescent images of mature $P$. falciparum LS parasites were captured at day 7 of infection. The merozoites were localized with antibodies to MSP1. The PVM was localized with antibodies to EXP-1. DNA was visualized with DAPI, and differential interference contrast microscopy images of the liver sections were captured. (A) Appearance of a budding merosome (white arrow, MSP1 panel) is associated with a perturbation in the membrane surrounding the mature LS (white arrow, DIC panel). (B and C) Merosomes adjacent to mature LS parasites (white arrows, MSP-1 panels). Note that the DIC image in B suggests that the merosome is ensconced within a membrane (white arrow, DIC panel and in the magnification shown in the lower right of the panel). The DIC image inset in B shows that the membranes of the mature LS and the membrane of the merosome have completely separated. (D) Unorganized merozoite masses appear to be spilling into the surrounding liver tissue, indicating that merozoite release occurs not only in merosomes. Note that individuated merozoites are visible. (E) A mature LS with multiple merozoite release events (white arrows, MSP1 panel) shows that the PVM has broken down (31), resulting in the presence of a small EXP-1-positive PVM remnant (white arrow, EXP-1 panel). Scale bars: $10 \mu m$.

not (Supplemental Figure 1A; supplemental material available online with this article; doi:10.1172/JCI62684DS1). Furthermore, at day 7, PTEX150 (28) expression and EXP-2 expression showed a partially overlapping staining pattern (Supplemental Figure 1B). Late LS development is marked by the expression of MSP1 and subsequent formation of merozoites, with MSP1 expression localized to the merozoite surface. Indeed, at day 7 after infection, there was robust expression of MSP1 (Figure 1D). The pattern of
MSP1 staining was indicative of cytomere formation, where multiple invaginations of the parasite plasma membrane eventually lead to the pinching off of membrane around each nascent exoerythrocytic merozoite. In contrast with the MSP1 staining, the PVM marker EXP-1 could clearly be seen to delineate the confines of the LS parasite where cytomere formation was still taking place (Figure 1D). All day 7 LS examined showed robust expression of MSP1, and in highly humanized parts of the liver substantial 
A

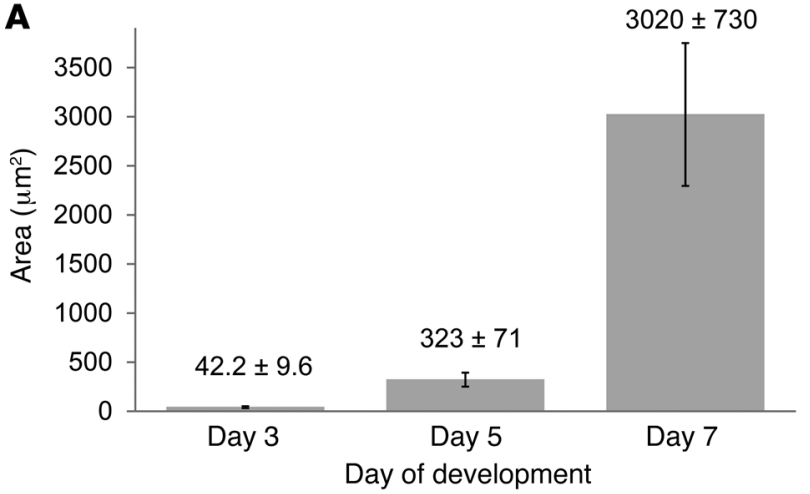

B

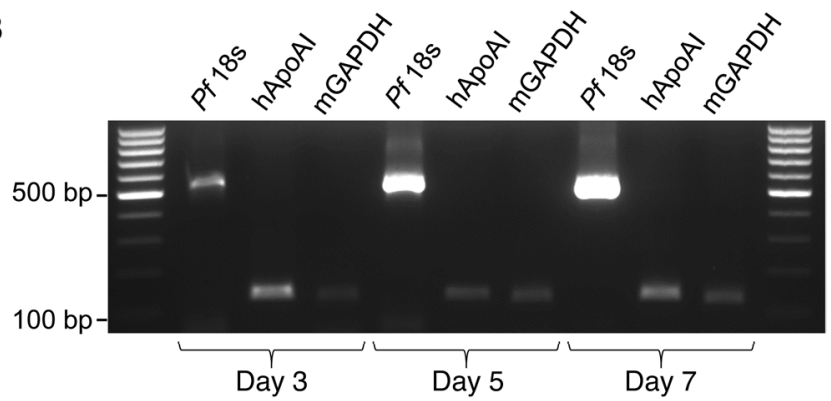

C

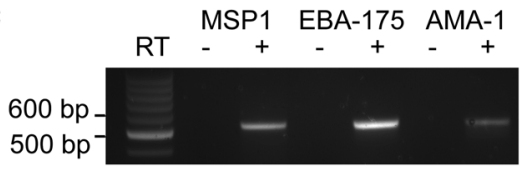

numbers of LS were observed (Figure 1E). IFA analysis also demonstrated the lack of MSP1 expression in 3 and 5 day LS and the lack of CSP expression in 7 day LS (data not shown).

Previous studies using intravital microscopy of fluorescent rodent malaria LS (both P. berghei and P. yoelii) have shown that merozoites are frequently released from infected hepatocytes as merosomes: packets of multiple merozoites held together by a surrounding host hepatocyte-derived membrane $(9,29,30)$. The rodent malaria merosomes bud off into the sinusoidal lumen and are carried in the bloodstream to the lung, where they burst, releasing erythrocyte-infectious merozoites (29). However, it remains unknown whether this key feature of late LS development is conserved in human malaria parasites. Here, we observed, for what we believe is the first time, budding of P. falciparum exoerythrocytic merozoite aggregates at day 7 after infection (Figure 2A), suggesting that merosome formation is conserved in human malaria parasites and can be modeled in the FRG huHep mouse model. Individual, fully segregated merosomes that appeared to be surrounded by host hepatocyte-derived membrane were observed (Figure 2B), and individual merozoites were visualized within the mature LS as well as within the merosomes (Figure 2C). Merosomes sometimes stayed in close proximity to the mature LS and appeared to retain a narrow connection (Figure 2C). However, often, less organized release of merozoites from late LS into the adjacent tissue was seen, and these releases contained individual merozoites (Figure 2D and Supplemental Figure 2). The full maturation of rodent malaria parasite LS in vitro and in vivo was shown to be accompanied by the breakdown of the PVM $(29,31)$, and we also saw this

\section{Figure 3}

LS growth and parasite gene expression in infected FRG huHep mice. (A) LS size was measured based on indirect immunofluorescence analysis of infected liver sections using the maximal diameters of parasites at 3, 5, and 7 days after infection. At least 14 LSs were analyzed for each time point and the results represented by LS parasite area. Data represent mean \pm SD. (B) RT-PCR on RNA isolated from infected FRG huHep livers demonstrates transcription of hapoAI, mGAPDH, and $P$. falciparum 18S rRNA (Pf 18S) at 3, 5, and 7 days after infection with sporozoites. (C) Transcripts for the parasite merozoite-stage proteins MSP1, EBA-175, and AMA-1 are detected in LS at day 7 after infection $(+)$ and are not present in the minus reverse transcriptase control (-). A 100-bp DNA ladder was run in the far left lanes of the gels as shown in $\mathbf{B}$ and $\mathbf{C}$, and pertinent fragment sizes are shown to the left of the gel images.

process in P. falciparum LS. This was indicated by the observation that the PVM marker EXP-1 in a fully mature LS labeled only a small PVM remnant (Figure 2E and Supplemental Figure 3).

$L S$ growth and gene transcription in the FRG buHep mouse. To further investigate LS growth in FRG huHep infections, the maximum area of multiple LS cross sections $(n \geq 14)$ was determined (Figure $3 \mathrm{~A}$ ). In day 3 infections, maximum LS diameter ranged from 5.0 to $8.4 \mu \mathrm{m}$, and the maximum LS area was $42.2 \pm 9.6 \mu \mathrm{m}^{2}$; at day 5, maximum LS diameter ranged from 17 to $26 \mu \mathrm{m}$, and the maximum LS area was $322 \pm 71 \mu \mathrm{m}^{2}$; and finally at day 7 , maximum LS diameter ranged from 50 to- $80 \mu \mathrm{m}$, and the maximum LS area was $3020 \pm 730 \mu \mathrm{m}^{2}$. These measurements demonstrate the remarkable growth acceleration during the final 2 days of LS development as well as the relative uniformity of LS schizont size at distinct developmental time points; the SD as a percentage of the mean was similar throughout development. Importantly, growth-retarded P. falciparum LSs, as often seen in in vitro culture, were never observed in day 7 FRG huHep infections.

To amplify P. falciparum transcripts using RT-PCR, RNA was isolated from infected liver tissue, reverse transcribed, and subject to amplification with oligonucleotide primers specific for human apoAI (hapoAI), mouse GAPDH (mGAPDH), and P. falciparum 18S rRNA. Parasites were detectable by RT-PCR at all time points of development, and amplification of transcribed parasite 18S rRNA was greatest in day 7 infections compared with either day 5 or day 3 infections, proportional to the massive increase in parasite biomass (Figure 3B). To further analyze LS maturation, we amplified transcripts for 3 genes indicative of merozoite maturation: MSP1, erythrocyte-binding antigen-175 (EBA-175), and apical membrane antigen-1 (AMA-1) (Figure 3C) at day 7 of infection. This is encouraging in that it not only implies that LS infection rates are high enough in the FRG huHep mice to produce sufficient transcript for amplification from whole infected tissue, but also that stage-appropriate transcripts are being expressed during LS maturation.

Ex vivo visualization of fluorescent $P$. falciparum LSs in the FRG buHep mouse. Previous analyses of rodent malaria LS transcriptomes and proteomes have relied on FACS of fluorescent parasites from hepatocytes isolated from perfused, collagenase-treated, infected livers (10). P. falciparum LS transcriptomic and proteomic analyses would be invaluable for research into this life-cycle stage, and the FRG huHep mouse could be utilized for FACS of fluorescent P. falciparum LS. A fluorescent P. falciparum 3D7 transgenic parasite was recently created that expressed GFP under control of the EF1 $\alpha$ 

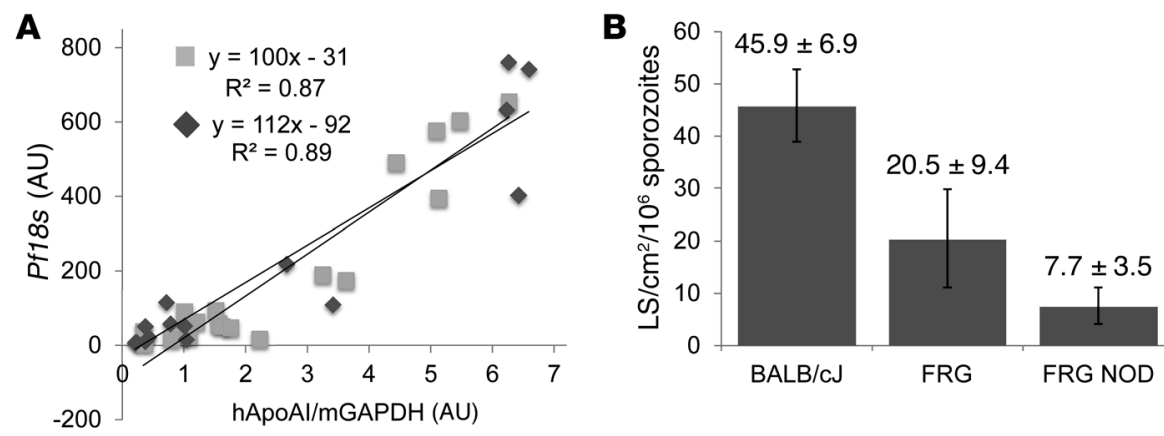

\section{Figure 4}

Correlation of LS burden with liver humanization in FRG huHep mice and comparison of LS density in P. falciparum-infected FRG huHep mice, FRG NOD huHep mice, and P. yoelii-infected BALB/cJ mice. (A) Liver tissue fragments (each point on the graph represents a single sample) taken from a 7-day LS infection of 2 FRG huHep mice (female littermates who received the same human donor hepatocytes) were analyzed by qRT-PCR for P. falciparum 18S rRNA burden (Pf 18S, arbitrary units) as well as the level of humanization based on the ratio of hapoAI transcripts relative to $\mathrm{mGAPDH}$ transcripts (arbitrary units). The results show a statistically significant, linear relationship (coefficient of determination, $R^{2}=0.87-0.89$ ) between LS burden and liver humanization in the 2 mice. (B) The level of $P$. falciparum LS burden in the FRG huHep mouse was compared with that of the FRG NOD huHep mouse and $P$. yoelii rodent malaria LS burden in BALB/cJ mice. LS burden is shown as LS/cm² $50-\mu \mathrm{m}$ liver section $/ 10^{6}$ sporozoites injected. Average LS counts per liver section were determined by analyzing at least 6 nonserial $50-\mu \mathrm{m}$ liver sections from 3 individual mice. Humanized mice had huHep repopulation levels above $80 \%$. The results show that the FRG huHep and FRG NOD huHep mice support robust $P$. falciparum LS infections. Data for $B$ represent mean \pm SD.

promoter throughout the life cycle (32). However, the P. falciparum $3 \mathrm{D} 7$ parasite line is not a robust gametocyte producer. Therefore, we recreated the transgenic parasite in the P. falciparum NF54 parasite line. Blood stages, oocysts, and salivary gland sporozoites from this line (NF54HT-GFP) were fluorescent (data not shown). NF54HT-GFP sporozoites (2 million) were injected into a FRG huHep mouse, and 6 days later, the mouse was sacrificed. The liver was removed, and lobes were immediately sectioned ex vivo without fixation and analyzed by fluorescent microscopy. GFP-fluorescent LS parasites were readily visualized (Supplemental Figure 4A). IFA following fixation and sectioning of liver lobes also showed the robust expression of GFP in the day 6 LS (Supplemental Figure 4B).

Quantification of P. falciparum LS burden in the FRG buHep mouse and the level of LS infection in the FRG buHep and FRG NOD buHep mice. We tested to determine whether the level of huHep repopulation within the FRG huHep mouse liver correlated with LS burden. We reasoned that the higher the repopulation, the larger the LS burden would be in any one part of the liver. Thus, on the same day, we injected 2 FRG huHep mice with approximately $80 \%$ repopulation with equal numbers of $P$. falciparum sporozoites ( 4 million) and sacrificed the mice at 7 days after injection. The mice were littermates and had received the same donor hepatocytes on the same day, but varied in their total human albumin levels. Liver sections (between 16 and 21) were taken from multiple lobes, and RNA was isolated from each individual section. After DNase treatment and reverse transcription, equal amounts of cDNA from each section were subjected to quantitative RT-PCR (qRT-PCR) analysis of parasite burden (based on parasite 18S rRNA transcription) and humanization (based on a ratio of hapoAI transcripts to $\mathrm{mGAPDH}$ transcripts). This allowed us to plot LS biomass with the extent of humanization. LS burden directly correlated in a linear fashion to the degree of humanization in a given sample of liver tissue (Figure 4A). The results also showed that once repopulation falls below a certain threshold, LS burden is undetectable, as demonstrated by a positive, non-zero X-intercept. Moreover, the slope of the best-fit line was similar for the 2 mice, suggesting reproducibility.
We next set out to determine the P. falciparum LS infection densities in the FRG huHep mice and FRG NOD huHep mice and compared them with a rodent malaria/mouse combination - the P. yoelii parasite in the BALB/cJ mouse. Nonserial $50-\mu \mathrm{m}$ liver slices were analyzed by IFA for LS numbers, and these numbers were related to the areas of the liver slices and the numbers of sporozoites injected. Calculations were made on 3 FRG huHep and FRG NOD huHep mouse livers (with huHep repopulation levels above $80 \%$ ) assayed at 7 days after injection of between 2 and 4 million $P$. falciparum sporozoites. The FRG huHep mouse was approximately $50 \%$ as susceptible to a $P$. falciparum LS infection $\left(21 \mathrm{LS} / \mathrm{cm}^{2}\right.$ $50-\mu \mathrm{m}$ liver section $/ 10^{6}$ sporozoites injected) as the BALB/cJ mouse was to $P$. yoelii LS infection ( $46 \mathrm{LS} / \mathrm{cm}^{2} 50-\mu \mathrm{m}$ liver section $/ 10^{6}$ sporozoites injected), whereas LS infection in the FRG NOD huHep mouse, although robust, was lower $\left(8 \mathrm{LS} / \mathrm{cm}^{2} 50-\mu \mathrm{m}\right.$ liver section $/ 10^{6}$ sporozoites injected) (Figure 4B).

Complete P. falciparum LS development in the FRG NOD buHep mouse and transition from LS-to-blood-stage infection. Demonstrating the completion of P. falciparum LS development with formation of infectious exoerythrocytic merozoites and the subsequent transition to blood-stage infection is an important next step. Following this transition in an animal model would further aid studies into the biology of late LS and exoerythrocytic merozoite infection of hurbc. Ultimately it might even allow for conducting parasite genetic crosses in a combined huHep/hurbc model. However, severe challenges have been encountered in the creation of immunocompromised mice that can support human hematopoietic development through the xenotransplantation of human hematopoietic stem cells, and this is especially true for erythrocyte development (33). The C57BL/6 background of the FRG huHep mouse does not support engraftment with hurbc, and this is partly due to the incompatibility of mouse macrophage-expressed signal regulatory protein $\alpha(\operatorname{SIRP} \alpha)$ with human CD47, which leads to the rapid clearance of hurbc by mouse macrophages (33, 34). A mutation in the SIRP $\alpha$ gene in the NOD mouse prevents this incompatibility, and thus, clearance of hurbc is reduced. We thus injected 

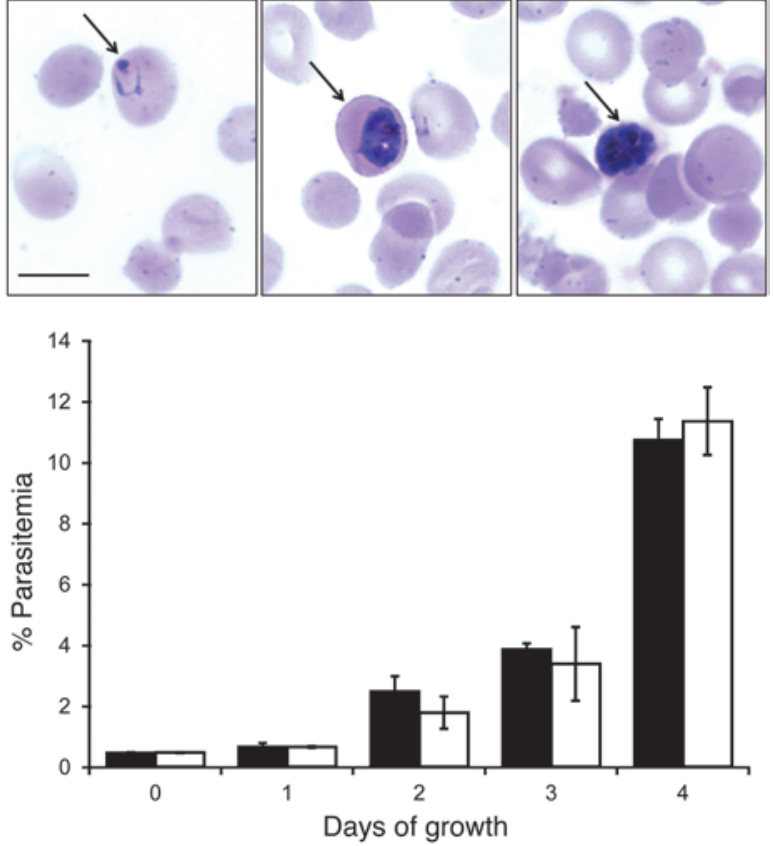

FRG NOD huHep mice with $P$. falciparum sporozoites to ensure they were able to support complete LS development. Indeed, at 7 days after sporozoite injection, mature LSs were seen in the livers of the mice, comparable to those observed in FRG huHep mice (Supplemental Figure 5).

We next explored whether the transition of the $P$. falciparum LS infection to a blood-stage infection in the FRG NOD huHep mouse is possible. Six days after sporozoite injection, FRG NOD huHep mice were injected intravenously with hurbc and again on day 7 after sporozoite injection. Blood was removed from the mouse by cardiac puncture at day 7 . The buffy coat was removed, and the blood was washed with standard in vitro $P$. falciparum culture medium, supplemented with an equal volume of hurbc, and cultured at $4 \%$ hematocrit. In 2 independent experiments with a total of 4 FRG NOD huHep mice, blood-stage parasites were consistently detected by Giemsa-stained thin blood smears from 1 to 5 days after the initiation of the in vitro culture. The parasites were subsequently maintained in continuous in vitro culture, and growth rates (Figure 5) were comparable to those of the parent NF54 strain used for mosquito infections and sporozoite production. In addition, the mouse-derived asexual parasite cultures successfully converted to produce gametocytes (Supplemental Figure $6 \mathrm{~A})$, which when fed to mosquitoes, resulted in the production of oocysts (Supplemental Figure 6B). Furthermore, salivary gland sporozoite numbers between the NF54 strain routinely used for our mosquito infections and the humanized mouse-transitioned NF54 parasites were similar (data not shown).

\section{Discussion}

We have shown here that FRG huHep mice support complete development of $P$. falciparum LS. Furthermore, we have shown that LS development in the FRG NOD huHep mouse culminated in the release of exoerythrocytic merozoites that invaded hurbc and initiated sustainable asexual erythrocytic replication in vitro, an achievement that, to date, has not been documented. The robust,

\section{Figure 5}

$P$. falciparum LS infection in FRG NOD huHep mice transitions to blood-stage infection. Growth of blood-stage P. falciparum parasites in in vitro culture that were obtained from infected FRG NOD huHep mice 7 days after sporozoite injection is shown. Infected mice were injected with hurbc on day 6 and 7 after sporozoite injection to allow asexual erythrocytic infection. Parasite-infected blood was removed from the mice and placed in in vitro rbc culture. Humanized mouse infection-derived asexual blood-stage parasites from 3 individual FRG NOD huHep mice (white bars) and parent NF54 parasites (black bars) were assayed for growth over 4 days in triplicate. Giemsa-stained thin blood smears were assayed for percentage of parasitemia and also to demonstrate the presence of healthy parasites in the culture (inset, left panel, ring stage; middle panel, trophozoite; right panel, schizont). Black arrows point to infected cells. Blood-stage parasites derived from sporozoite-induced FRG NOD huHep mouse infections show normal in vitro growth characteristics. Scale bar: $10 \mu \mathrm{m}$. Data represent mean \pm SD. reproducible $P$. falciparum LS infection in FRG huHep and FRG NOD huHep mice will greatly accelerate studies on human malaria LS. Furthermore, that the parasites transition from LS to blood stage in a small animal model is, we believe, unprecedented and will allow studies into the liver-to-blood transition of human malaria parasites, epigenetics of the parasite throughout the life cycle, and other applications, such as conducting P. falciparum genetic crosses. Such crosses are currently only possible in chimpanzees, and thus few have been carried out (35). Although direct comparisons with the SCID Alb-uPA huHep mouse were not made in our study, the robustness of the FRG huHep mouse models and their ability to support reproducible transition from LS to a normal in vitro bloodstage infection point to substantial advantages of these models.

Immunocompromised mice into which hurbc are continuously injected are able to support P. falciparum blood-stage infections, but the drawbacks are that the mice either have to be infected with adapted strains of $P$. falciparum (36) or continuously treated with clodronate liposomes to deplete macrophages (37). If the FRG NOD huHep mouse was also used for hurbc reconstitution, the clodronate depletion of the resident macrophages in the liver, the Kupffer cells, might have an effect on liver infection by sporozoites. A mouse reconstituted with a human hematopoietic system that produced its own hurbc would be ideal, but such models currently do not support stable hurbc production and maintenance (21).

Evidence for the robustness of the FRG huHep models and their utility for revealing new biological features of $P$. falciparum LS is based on growth observations of LS parasites at 3, 5, and 7 days after infection with $P$. falciparum sporozoites. LS expressed CSP at 3 and 5 days of development, but ceased CSP expression late in development. We observed that the known blood-stage PV marker EXP-2 $(26,27)$ was expressed throughout LS development. Importantly, EXP-2 was recently shown to be a critical component of the PV translocon of exported proteins in P. falciparum blood stages where EXP-2 constitutes the potential pore through which parasite proteins destined for the erythrocyte cytoplasm or erythrocyte 
surface pass (28). It is currently unknown whether LSs employ a similar export mechanism, but our findings indicate that the poreforming component of the translocon is expressed in LS as well as a further component of the translocon, PTEX150. Thus, it is likely that the translocon is not unique to blood-stage parasites and in LS could serve to transport proteins into the infected hepatocyte cytoplasm. CSP was shown to be exported in rodent malaria LS (38), but we have, to date, seen no evidence for CSP export in the FRG huHep mouse infections. Thus, further work is needed to explore whether the PVM translocon is active in P. falciparum LS.

At day 7 of P. falciparum LS development, breakdown of the PVM occurred, as visualized by the disappearance of circumferential EXP-1 expression. PVM breakdown was previously shown to be a critical step in rodent malaria models preceding merozoite release (29-31). Furthermore, MSP1 protein expression as well as transcript abundance of the maturing merozoite markers MSP1, EBA175 , and AMA- 1 at 7 days of development suggested that complete LS development had occurred. Indeed, LS parasites were visualized that contained differentiated exoerythrocytic merozoites, each with individual nuclei. We also observed, for what we believe is the first time, the formation of merosomes in 7-day-old LSs, suggesting that $P$. falciparum shares this important feature with rodent malaria parasites for the delivery of merozoites to the bloodstream $(9,29,30)$. The merosome is thought to prevent phagocytosis of the merozoites as they leave the host hepatocyte and protect the merozoites as they journey to the bloodstream before finally being released in the pulmonary vasculature, whereupon they invade rbc (29). Thus, the FRG huHep and FRG NOD huHep mice support the entire development of $P$. falciparum LS parasites, allowing accurate modeling of human preerythrocytic malaria parasite infections.

The relatively synchronous development and the immense biomass of the late LS parasites seen in the FRG huHep mice, along with our evidence for exoerythrocytic merozoite formation and merosome release, shows how superior this in vivo model of development is compared with current available in vitro models. The reported sizes of late $P$. falciparum LSs obtained from in vitro culture range from only 15 to $40 \mu \mathrm{m}$ in diameter $(6,7,39,40)$, whereas we visualized LS sizes up to $80 \mu \mathrm{m}$ in diameter, which, based on previous volume calculations (29), contain between 40,000 and 60,000 merozoites. A small number of studies on $P$. falciparum LS development in humans have been undertaken $(41,42)$, and the size of 6 day LS ranged from 55 to $60 \mu \mathrm{m}$. A more complete microscopic study of mature P. falciparum LS in the chimpanzee, which is susceptible to both P. falciparum preerythrocytic and blood-stage infections, has also been carried out, showing late LS measured from 60 to $100 \mu \mathrm{m}$ in diameter (43). Thus, our studies show that LS development in the FRG huHep mice takes place with the dynamics and biomass amplification comparable to those seen in both human and chimpanzee infections. Furthermore, in vitro studies of $P$. falciparum LS development in primary hepatocytes have observed numerous small, growth-stunted LS forms up to 11 days after infection (44). We never observed growth-stunted LS in the FRG huHep mice, indicating that in vitro development leads to LS artifacts (both depressed growth rate and presence of small LS forms) that are not found in in vivo infections. Since LS development appears to occur in a physiologically relevant manner and synchronously, the FRG huHep mouse should be an excellent model for studying the effect of interventions on LS developmental progression. To support this, we have shown that LS burden is reproducibly quantifiable. This will enable quantitative analysis of drug efficacy in elimination of LS. The use of the FRG huHep mouse for assessing drug efficacy against LS is an attractive path, as it is more likely to correspond to actual clinical efficacy in humans. Drugs that target the P. falciparum LS will be metabolized in huHeps and the treatment will measure in vivo effects against the relevant human parasites.

The LS-to-blood-stage transition in the FRG NOD huHep mouse infections will also be useful in the analysis of $P$. falciparum genetically attenuated strains (45). Immunizations with genetically attenuated rodent malaria parasites that arrest in LS development induce potent sterilizing immune responses and completely protect against a subsequent wild-type sporozoite challenge (46), providing the rationale for testing attenuated $P$. falciparum strains in human vaccination (47). Work using the SCID Alb-uPA huHep mouse showed that a first generation genetically attenuated $P$. falciparum, the $p 52^{-} p 36^{-}$double-gene knockout (19), arrested early in LS development, similar to its P. yoelii counterpart (48), but questions remain as to the sensitivity of this model for detecting rare parasites that exhibit some growth and can lead to blood-stage infection. The FRG NOD huHep mouse might constitute a more sensitive model that can be used for such studies, since the LS-toblood-stage transition is achievable. This is particularly relevant when rodent malaria parasites cannot be used to evaluate gene knockouts; the $P$. falciparum genome contains many genes that are not present in rodent malaria species.

In summary, we have demonstrated that robust, biologically relevant $P$. falciparum LS development occurs in the FRG huHep and FRG NOD huHep mouse models, and the transition to bloodstage infection is possible in the FRG NOD huHep mouse. These in vivo models of $P$. falciparum preerythrocytic infection are useful for the study of drug interventions, parasite attenuation, and even innate immune responses to LS infection. This enables a great expansion of LS research with malaria parasites that infect humans. Equally important, the models could well provide unique opportunities for in vivo studies of $P$. vivax malaria, particularly the relapsing hypnozoite stages of this parasite.

\section{Methods}

FRG huHep and FRG NOD buHep mice. Female FRG huHep (on the C57BL/6 background) (22) and FRG NOD huHep mice with human chimeric livers were purchased from Yecuris Corp. Backcrossing the FRG mouse (which is on the C57BL/ 6 background) to the NOD ShiLtJ mouse created the FRG NOD mouse. The FRG NOD mouse is as healthy as the FRG mouse, breeds as a homozygote, and is equally permissive to huHep chimerism as the FRG mouse. The FRG huHep and FRG NOD huHep mice were both susceptible to $P$. falciparum LS stage development (Yecuris Corp.).

All mice had human repopulation levels above $60 \%$, and the majority of the mice used for this study had repopulation levels above $80 \%$, which we found supported robust development of $P$. falciparum LS. Repopulation levels are estimated based on the human serum albumin levels of the mouse, and over 100 animals have been used to correlate human serum albumin levels with human repopulation. To determine human serum albumin levels, blood samples from each repopulated mouse were collected via the saphenous vein and analyzed using the Human Albumin ELISA Quantitation Set (Bethyl Laboratories) according to the manufacture's protocol, with slight modifications. We have determined that to achieve saturable binding and reproducible results, the incubation time for the binding of the standards and unknowns to the capture antibody must be increased from 1 to 2 hours. All other steps were performed as described in the manufacturer's protocol. To determine the repopulation 
levels based on the results of the Human Albumin ELISA Quantitation Set, hepatocytes were isolated from FRG huHep and FRG NOD huHep mice by perfusion using collagenase and low-speed centrifugation, and then repopulation was measured in 1 of 2 ways. In the first method, the isolated hepatocytes were plated on collagen-coated dishes at $0.285 \times 10^{6} \mathrm{cells} / \mathrm{cm}^{2}$ and incubated at $37^{\circ} \mathrm{C}$ in $5 \% \mathrm{CO}_{2}$ for $20-24$ hours. The cells were rinsed twice with PBS, $\mathrm{pH} 7.2$, and fixed for 10 minutes at room temperature with $4 \%$ paraformaldehyde. After washing 3 times with PBS, $\mathrm{pH} 7.2$, to remove the paraformaldehyde, the cells were blocked and permeabilized in PBS, $\mathrm{pH} 7.2$, containing $0.1 \%$ Triton X-100 (v/v), and $0.25 \%(\mathrm{v} / \mathrm{v})$ normal goat serum for 1 hour at room temperature. The hepatocytes were stained overnight with a rabbit polyclonal FAH antibody (specific to the huHeps) and a mouse hepatocyte-specific rat monoclonal antibody in blocking buffer. The cells were washed 3 times with PBS, pH 7.2, and the primary antibodies were detected using goat anti-rabbit Alexa Fluor 555 and donkey anti-rat Alexa Fluor 488 antibodies (Life Technologies). To identify all cells, a DNAspecific Hoechst dye was added to the secondary antibody solution. Images of 5-6 fields for each fluorophore were captured using an EVOS fluorescent digital inverted microscope, and the numbers of cells were determined using ImageJ64 software. Human levels of repopulation were determined by dividing the FAH-positive cells by the total number of cells (FAH positive and mouse hepatocyte positive). In the second method for assessing repopulation, we used flow cytometry on hepatocytes isolated from FRG huHep and FRG NOD huHep mice to determine the repopulation indices. Briefly, approximately 400,000 hepatocytes isolated from a mouse were incubated with a human HLA ABC biotin-conjugated antibody (eBioscience) and a cocktail of mouse hepatocyte-specific rat monoclonal antibodies (Oregon Stem Cell Center) for 1 hour on ice. The cells were washed and incubated in streptavidin-conjugated with APC (BD Biosciences) and goat anti-rat Alexa Fluor 555 for 1 hour on ice. Once again, the cells were washed and brought up in buffer containing propidium iodine to distinguish live from dead cells. The cells were analyzed on a BD FACSCalibur analyzer and the data evaluated using FlowJo software to determine the percentage of human versus mouse hepatocytes. To date, we have analyzed over 100 FRG huHep and FRG NOD huHep mice for human repopulation using either or both of these methods. When the 2 methods were compared, they gave comparable results. With the data collected, we have determined the following range of repopulation based on human albumin levels in the mouse: human albumin of $2.0-3.0 \mathrm{mg} / \mathrm{ml}, 50 \%-70 \%$ repopulation; $3.0-4.0 \mathrm{mg} / \mathrm{ml}, 70 \%-90 \%$ repopulation, and above $4.0 \mathrm{mg} / \mathrm{ml}$, more than $90 \%$ repopulation. We realize that our correlation differs from those published by Bissig et al. (25), but we believe that with the ELISA protocol optimization we have implemented and the number of FRG huHep and FRG NOD huHep mice we have evaluated, our correlation of human albumin to replacement indices in the FRG huHep and FRG NOD huHep mice is accurate.

Mice used in the study were cycled on and off NTBC on a 2-week schedule. In week 1, mouse water was supplemented with NTBC at $16 \mathrm{mg} / \mathrm{l}$ for 3 days, $0.8 \mathrm{mg} / 1$ for 2 days, and then $0.2 \mathrm{mg} / 1$ for 2 days. The drug was then removed for week 2 . This schedule continued until the end of the experiment.

P. falciparum sporozoite production. A. stephensi mosquitoes (originating from the Walter Reed Army Institute of Research, Silver Spring, Maryland, USA) were maintained at $27^{\circ} \mathrm{C}$ and $75 \%$ humidity on a 12 -hour light/12hour dark cycle. Larval stages were reared following standard protocols as described in the MR4 manual, with larval stages maintained on finely ground Tetramin fish food and adult mosquitoes maintained on $8 \%$ dextrose in $0.05 \%$ para-aminobenzoic acid (PABA) water.

In vitro $P$. falciparum NF54 blood-stage cultures were maintained in RPMI 1640 (25 mM HEPES, $2 \mathrm{mM}$ L-glutamine) supplemented with $50 \mu \mathrm{m}$ hypoxanthine and $10 \% \mathrm{~A}^{+}$human serum in an atmosphere of $5 \% \mathrm{CO}_{2}, 5 \%$ $\mathrm{O}_{2}$, and $90 \% \mathrm{~N}_{2}$. Cells were subcultured into $\mathrm{O}^{+}$erythrocytes. Gametocyte cultures were initiated at $5 \%$ hematocrit and $0.8 \%-1 \%$ parasitemia (mixed stages) and maintained for up to 17 days with daily medium changes.

Non-blood fed adult female mosquitoes 3 to 7 days after emergence were fed on gametocyte cultures. Gametocyte cultures were quickly spun down and the pelleted infected erythrocytes diluted to a $40 \%$ hematocrit with fresh $\mathrm{A}^{+}$human serum and $\mathrm{O}^{+}$erythrocytes. Mosquitoes were allowed to feed through Parafilm for up to 20 minutes. Following blood feeding, mosquitoes were maintained for up to 19 days at $27^{\circ} \mathrm{C}, 75 \%$ humidity, and provided with $8 \%$ dextrose solution in PABA water. Infection prevalence was checked at days $7-10$ by examining dissected midguts under light microscopy for the presence of oocysts with salivary gland dissections performed at days 14-19.

Creation of a P. falciparum NF54 line expressing GFP throughout the life cycle. A $P$. falciparum 3D7 transgenic parasite line expressing GFP under the control of the EF1 $\alpha$ promoter has previously been created (32). The parasite line, named 3D7HT-GFP, expresses GFP throughout the life cycle under the constitutive EF1 $\alpha$ promoter, and the transgene is integrated into the dispensable $P f 47$ locus. We wanted to create the same transgenic parasite in P. falciparum NF54, since gametocyte production in this line is more robust. Thus, the same plasmid used to create the 3D7HT-GFP line (pEFGFP) was transfected into P. falciparum NF54 to create NF54HT-GFP (Supplemental Figure 7A). Briefly, The NF54 line P. falciparum parasites were synchronized at ring stage with sorbitol 2 days prior to transfection. Transfection of P. falciparum ring stages with $100 \mu \mathrm{g}$ of DNA was performed by electroporation at $0.31 \mathrm{kV}$ and $950 \mu \mathrm{F}$ with a Bio-Rad Gene Pulser (Bio-Rad). Cultures were placed on the positive selection drug WR99210 (Jacobus Pharmaceuticals) 6 hours after transfection and maintained in the culture at a final concentration of $5 \mathrm{nM}$. Drug-resistant parasites were subjected to successive rounds of on/off drug selection, and integration was confirmed by PCR. Individual clones of the NF54HT-GFP line were isolated by limiting dilution, and genotypic analysis was confirmed by PCR using the same primers (Supplemental Table 1) and methodology used to create 3D7HT-GFP (ref. 32 and Supplemental Figure 7B). This NF54HT-GFP line was used solely to demonstrate ex vivo LS GFP fluorescence and complete LS development.

In vivo sporozoite infection and liver isolation. Mice were injected intravenously into the tail vein with between 1 and 4 million P. falciparum NF54 sporozoites in $100 \mu \mathrm{l}$ of RPMI medium isolated from the salivary glands of infected mosquitoes. Mice were euthanized at 3, 5, 6, and 7 days after sporozoite infection. The liver was perfused with PBS through the hepatic portal vein, removed, and separated into lobes. Small pieces of liver from each lobe were added to Trizol (Invitrogen), homogenized, and stored at $-80^{\circ} \mathrm{C}$ until used for RNA extraction. The remaining lobes were fixed in $4 \%$ electron microscopy grade formaldehyde in PBS, which was replaced by TBS plus $0.05 \%(\mathrm{w} / \mathrm{v})$ sodium azide after 24 hours. The fixed lobes were subsequently sliced into $50-\mu \mathrm{m}$ sections for IFA, as detailed previously (12).

Indirect IFA of P. falciparum LSs. IFA was carried out as previously described (12) on liver sections isolated from mice with 3, 5, 6, and 7 day LS development. huHeps were detected with a rabbit anti-FAH antibody (a gift from Yecuris Corp.). P. falciparum LS were detected with a CSP mouse monoclonal antibody, an EXP-2 mouse monoclonal antibody (27), a rabbit antibody to PF10_0164 (14) and a mouse monoclonal MSP1 antibody (MR4, ATCC), a rabbit antibody to PTEX150 antibody, and a rabbit antibody to EXP-1.

qRT-PCR. All oligonucleotide primers used in this study are detailed in Supplemental Table 1. Total RNA from liver lobe samples was extracted using Trizol (Invitrogen) and DNase treated using Turbo-DNA Free (Ambion). First-strand cDNA was synthesized from RNA using the Superscript III Platinum RT Kit (Invitrogen). The resulting cDNA was used for the amplification of hapoAI, mGAPDH, and P. falciparum 18S rRNA, 
MSP1, EBA-175, and AMA-1 cDNA. qPCR was carried out with SYBR green (Invitrogen) using the Applied Biosystems 7300 Real-Time PCR System and associated software. Relative copy numbers for the transcripts under study were calculated using the $\Delta \Delta \mathrm{Ct}$ method. Each qPCR used identical quantities of first-strand cDNA generated from small sections of each of the liver lobes of 2 FRG huHep mice. The mice were littermates and received the same donor hepatocytes on the same date and were also injected with $P$. falciparum sporozoites (4 million) on the same date.

Measurement of LS infectivity in FRG buHep and FRG NOD buHep mice. The level of $P$. falciparum LS infectivity of the FRG huHep and FRG NOD huHep mice was compared with that of the commonly studied P. yoelii rodent malaria in the recipient BALB/cJ mouse and expressed as $\mathrm{LS} / \mathrm{cm}^{2}$ $50-\mu \mathrm{m}$ liver section $/ 10^{6}$ sporozoites injected. Average LS counts per liver section were determined by analyzing at least 6 nonserial $50-\mu \mathrm{m}$ liver sections from 3 individual mice (for the FRG huHep mice and FRG NOD huHep mice, all had human repopulation levels above $80 \%$ ) by IFA using antibody to MSP1. The LS counts were normalized against the number of sporozoites injected. The P. yoelii/BALB/cJ combination gave results that are similar to those seen for the $P$. berghei/C57BL/6 combination (9).

$L S$-to-blood-stage transition and in vitro culture of blood stages derived from FRG NOD huHep mouse infections. Four FRG NOD huHep mice were injected intravenously with $P$. falciparum sporozoites. Six days after injection, mice were injected intravenously $(400 \mu \mathrm{l})$ with packed $\mathrm{O}^{+}$hurbc. The intravenous injection was repeated on day 7. Two hours later, mice were sacrificed and blood was removed by cardiac puncture. The blood was added to $10 \mathrm{ml}$ complete medium (RPMI 1640 with 25 mM HEPES, 2 mM L-glutamine, $50 \mu \mathrm{M}$ hypoxanthine, and $10 \% \mathrm{~A}^{+}$human serum), pelleted by centrifugation at $200 \mathrm{~g}$, and the supernatant along with the buffy coat (containing white blood cells) removed. The rbc were then washed 3 times with $10 \mathrm{ml}$ complete medium, with pelleting and centrifugation as detailed above. After the third wash, an equal volume of packed $\mathrm{O}^{+}$hurbc (approximately $400 \mu \mathrm{l}$ ) was added, and the total rbc pellet was resuspended in complete medium to $2 \%$ hematocrit. Cultures were split equally into 6 wells of a 6-well plate and maintained in an atmosphere of $5 \% \mathrm{CO}_{2}, 5 \% \mathrm{O}_{2}$, and $90 \%$ $\mathrm{N}_{2}$. Cultures were fed daily and $50 \mu \mathrm{l}$ of fresh packed hurbc were added every 5 days to each well. Once parasitemia reached $1 \%$, serial dilutions of parasites were carried out to maintain healthy cultures.

Analysis of in vitro parasite growth in hurbc. Donor cultures were used at the greater than $94 \%$ ring stage to set up individual cultures at $0.5 \%$ parasitemia and $2 \%$ hematocrit in complete medium (RPMI 1640 with 25 mM HEPES, $2 \mathrm{mM}$ L-glutamine, $50 \mu \mathrm{M}$ hypoxanthine, and $10 \% \mathrm{~A}^{+}$human serum) in a 24-well plate format. Triplicate samples of each culture $(200 \mu \mathrm{l})$ were removed daily from individual wells for measurement of parasitemia. The rbc sample was pelleted by centrifugation at $200 \mathrm{~g}$, smeared onto glass slides, and Giemsa-stained for microscopic evaluation of the percentage of parasitemia.

Statistics. Data are shown as mean \pm SD. For linear regression modeling, the coefficient of determination, $R^{2}$, was defined using the GraphPad Prism program.

Study approval. The study was performed in strict accordance with the recommendations in the Guide for the Care and Use of Laboratory Animals of the NIH (8th edition. Revised 2011). To this end, the Seattle Biomedical Research Institute has an Assurance from the Public Health Service (PHS) through the Office of Laboratory Animal Welfare (OLAW) for work approved by its IACUC. The PHS Assurance number is A3640-01. All of the work carried out in this study was specifically reviewed and approved by the Seattle Biomedical Research Institute IACUC.

\section{Acknowledgments}

We would like to thank the insectary staff at Seattle BioMed for the culture of $P$. falciparum gametocytes and sporozoite production and Viswanathan Lakshmanan for help with $P$. falciparum bloodstage culture as well as the staff at Yecuris Corp. for all their guidance in the care and use of the FRG huHep mice and FRG NOD huHep mice. We are grateful to Brendan Crabb for the PTEX150 antibody, David Cavanagh for the EXP-2 antibody, Klaus Lingelbach for the EXP-1 antibody, and Robert Sinden for the pEFGFP plasmid used to create P. falciparum NF54HT-GFP. The current study was funded by grants awarded to S.H.I. Kappe from the Bill and Melinda Gates Foundation (OPP1016829) and the Department of Defense (W81XWH-11-2-0184).

Received for publication January 3, 2012, and accepted in revised form July 12, 2012.

Address correspondence to: Stefan H.I. Kappe, Seattle Biomedical Research Institute, 307 Westlake Avenue North, Seattle, Washington 98109, USA. Phone: 206.256.7205; Fax: 206.256.7229; E-mail: stefan.kappe@seattlebiomed.org.
1. WHO. World Malaria Report 2010. Geneva, Switzerland: World Health Organization; 2010.

2. Yamauchi LM, Coppi A, Snounou G, Sinnis P. Plasmodium sporozoites trickle out of the injection site. Cell Microbiol. 2007;9(5):1215-1222.

3. Mota MM, et al. Migration of Plasmodium sporozoites through cells before infection. Science. 2001;291(5501):141-144.

4. Vaughan AM, Aly AS, Kappe SH. Malaria parasite pre-erythrocytic stage infection: gliding and hiding. Cell Host Microbe. 2008;4(3):209-218.

5. Trager W, Jensen JB. Human malaria parasites in continuous culture. Science. 1976;193(4254):673-675.

6. Mazier D, et al. Complete development of hepatic stages of Plasmodium falciparum in vitro. Science. 1985;227(4685):440-442.

7. Sattabongkot J, et al. Establishment of a human hepatocyte line that supports in vitro development of the exo-erythrocytic stages of the malaria parasites Plasmodium falciparum and P. vivax. Am J Trop Med Hyg. 2006;74(5):708-715.

8. Lindner SE, Miller JL, Kappe SH. Malaria parasite pre-erythrocytic infection: preparation meets opportunity. Cell Microbiol. 2012;14(3):316-324.

9. Tarun AS, et al. Quantitative isolation and in vivo imaging of malaria parasite liver stages. Int $J$
Parasitol. 2006;36(12):1283-1293.

10. Tarun AS, et al. A combined transcriptome and proteome survey of malaria parasite liver stages. Proc Natl Acad Sci U S A. 2008;105(1):305-310.

11. Pei Y, et al. Plasmodium pyruvate dehydrogenase activity is only essential for the parasite's progression from liver infection to blood infection. $\mathrm{Mol}$ Microbiol. 2010;75(4):957-971.

12. Vaughan AM, et al. Type II fatty acid synthesis is essential only for malaria parasite late liver stage development. Cell Microbiol. 2009;11(3):506-520.

13. Mikolajczak SA, et al. Disruption of the Plasmodium falciparum liver-stage antigen-1 locus causes a differentiation defect in late liver-stage parasites. Cell Microbiol. 2011;13(8):1250-1260.

14. Mackellar DC, O'Neill MT, Aly AS, Sacci JB, JrCowman AF, Kappe SH. Plasmodium falciparum PF10_0164 (ETRAMP10.3) is an essential parasitophorous vacuole and exported protein in blood stages. Eukaryot Cell. 2010;9(5):784-794.

15. Morosan S, et al. Liver-stage development of Plasmodium falciparum, in a humanized mouse model. J Infect Dis. 2006;193(7):996-1004.

16. Sacci JB, et al. Plasmodium falciparum infection and exoerythrocytic development in mice with chimeric human livers. Int J Parasitol. 2006;36(3):353-360.
17. Meuleman P, et al. Morphological and biochemical characterization of a human liver in a UPA-SCID mouse chimera. Hepatology. 2005;41(4):847-856.

18. Mercer DF, et al. Hepatitis C virus replication in mice with chimeric human livers. Nat Med. 2001;7(8):927-933.

19. VanBuskirk KM, et al. Preerythrocytic, liveattenuated Plasmodium falciparum vaccine candidates by design. Proc Natl Acad Sci U S A. 2009;106(31):13004-13009.

20. Heckel JL, Sandgren EP, Degen JL, Palmiter RD, Brinster RL. Neonatal bleeding in transgenic mice expressing urokinase-type plasminogen activator. Cell. 1990;62(3):447-456.

21. Legrand $\mathrm{N}$, et al. Humanized mice for modeling human infectious disease: challenges, progress, and outlook. Cell Host Microbe. 2009;6(1):5-9.

22. Azuma H, et al. Robust expansion of human hepatocytes in $\mathrm{Fah}^{-/-} / \mathrm{Rag}^{-/-} / \mathrm{Il}_{2} \mathrm{rg}^{-/-}$mice. Nat Biotechnol. 2007;25(8):903-910.

23. Bissig KD, Le TT, Woods NB, Verma IM. Repopulation of adult and neonatal mice with human hepatocytes: a chimeric animal model. Proc Natl Acad Sci U S A. 2007;104(51):20507-20511.

24. Grompe $M$, et al. Pharmacological correction of neonatal lethal hepatic dysfunction in a murine 
model of hereditary tyrosinaemia type I. Nat Genet. 1995;10(4):453-460.

25. Bissig KD, et al. Human liver chimeric mice provide a model for hepatitis B and C virus infection and treatment. J Clin Invest. 2010;120(3):924-930.

26. Fischer K, et al. Characterization and cloning of the gene encoding the vacuolar membrane protein EXP-2 from Plasmodium falciparum. Mol Biochem Parasitol. 1998;92(1):47-57.

27. Johnson D, et al. Characterization of membrane proteins exported from Plasmodium falciparum into the host erythrocyte. Parasitology. 1994;109(pt 1):1-9.

28. de Koning-Ward TF, et al. A newly discovered protein export machine in malaria parasites. Nature. 2009;459(7249):945-949.

29. Baer K, Klotz C, Kappe SH, Schnieder T, Frevert U. Release of hepatic Plasmodium yoelii merozoites into the pulmonary microvasculature. PLoS Pathog. 2007;3(11):e171.

30. Sturm A, et al. Manipulation of host hepatocytes by the malaria parasite for delivery into liver sinusoids. Science. 2006;313(5791):1287-1290.

31. Graewe $S$, et al. Hostile takeover by Plasmodium: reorganization of parasite and host cell membranes during liver stage egress. PLoS Pathog. 2011;7(9):e1002224.

32. Talman AM, Blagborough AM, Sinden RE. A Plasmodium falciparum strain expressing GFP throughout the parasite's life-cycle. PLoS One. 2010;5(2):e9156.
33. Takenaka K, et al. Polymorphism in Sirpa modulates engraftment of human hematopoietic stem cells. Nat Immunol. 2007;8(12):1313-1323.

34. Hu Z, Van Rooijen N, Yang YG. Macrophages prevent human red blood cell reconstitution in immunodeficient mice. Blood. 2011;118(22):5938-5946.

35. Ranford-Cartwright LC, Mwangi JM. Analysis of malaria parasite phenotypes using experimental genetic crosses of Plasmodium falciparum. Int J Parasitol. 2012;42(6):529-534.

36. Jimenez-Diaz MB, et al. Improved murine model of malaria using Plasmodium falciparum competent strains and non-myelodepleted NODscid IL2Rgammanull mice engrafted with human erythrocytes. Antimicrob Agents Chemother. 2009;53(10):4533-4536

37. Arnold L, et al. Further improvements of the P. falciparum humanized mouse model. PLoS One. 2011;6(3):e18045.

38. Singh AP, et al. Plasmodium circumsporozoite protein promotes the development of the liver stages of the parasite. Cell. 2007;131(3):492-504.

39. Karnasuta C, et al. Complete development of the liver stage of Plasmodium falciparum in a human hepatoma cell line. Am J Trop Med Hyg. 1995;53(6):607-611.

40. Meis JF, et al. Fine structure of the malaria parasite Plasmodium falciparum in human hepatocytes in vitro. Cell Tissue Res. 1986;244(2):345-350.

41. Jeffery GM, Wolcott GB, Young MD, Williams D.
Exo-erythrocytic stages of Plasmodium falciparum. Am J Trop Med Hyg. 1952;1(6):917-925.

42. Shortt HE, Fairley NH, Covell G, Shute PG, Garnham PC. The pre-erythrocytic stage of Plasmodium falciparum. Trans $R$ Soc Trop Med Hyg. 1951;44(4):405-419.

43. Meis JF, et al. Plasmodium falciparum: studies on mature exoerythrocytic forms in the liver of the chimpanzee, Pan troglodytes. Exp Parasitol. 1990;70(1):1-11.

44. Dembele L, et al. Towards an in vitro model of Plasmodium hypnozoites suitable for drug discovery. PLoS One. 2011;6(3):e18162.

45. Vaughan AM, Wang R, Kappe SH. Genetically engineered, attenuated whole-cell vaccine approaches for malaria. Hum Vaccin. 2010;6(1):107-113.

46. Wang R, Smith JD, Kappe SH. Advances and challenges in malaria vaccine development. Expert Rev Mol Med. 2009;11:e39.

47. Khan SM, Janse CJ, Kappe SH, Mikolajczak SA. Genetic engineering of attenuated malaria parasites for vaccination [published online ahead of print May 3, 2012]. Curr Opin Biotechnol. doi:10.1016/j.copbio.2012.04.003.

48. Labaied M, Harupa A, Dumpit RF, Coppens I, Mikolajczak SA, Kappe SH. Plasmodium yoelii sporozoites with simultaneous deletion of P52 and P36 are completely attenuated and confer sterile immunity against infection. Infect Immun. 2007;75(8):3758-3768. 